\title{
Long-term survival with CML with imatinib or transplantation as first-line treatment: Comparison of outcomes from CML Studies IIIA and IV
}

\author{
Rüdiger Hehlmann \\ Mannheim Medical Faculty, Heidelberg University \\ Rüdiger Hehlmann, o. Prof. Dr. med. Dr. h. c., \\ Im Langgewann 45, 69469 Weinheim, Germany
}

\section{Summary}

With introduction of the tyrosine kinase inhibitor (TKI) imatinib, the treatment strategy of CML has profoundly changed. TKIs became the first-line treatment of choice for CML competing with allogeneic hematopoietic stem cell transplantation (HCT). Variables to be considered in choosing TKIs for first-line therapy are as follows: conventional risk score; cytogenetic findings with major-route additional chromosomal aberrations (ACA) at diagnosis, and high-risk ACA in the course of CML; comorbidities; treatment costs. In cases of refractoriness to imatinib, the $2^{\text {nd }}$ line treatment options are: clinical response milestones; adherence to therapy; resistance mutations; clonal evolution; therapy intolerance; drug safety; health care setting.

CML Study IV, a randomized treatment study concerning imatinib dose optimization and combined therapy with imatinib and cytarabine or interferon $\alpha$ included 1551 newly diagnosed patients in chronic phase. The key outcome was no superiority of survival of any treatment option. Imatinib $400 \mathrm{mg}$ provides close to normal life expectancy in chronic-phase CML patients.
E-mail: hehlmann.eln@gmail.com

Survival is independent of time to response. Outcome of CML is currently more determined by disease and patients' factors, e.g., comorbidities and smoking, and by center effects than by initial treatment selection. A comparison of long-term survival after HCT or imatinib treatment showed that low risk patients had similar survival with both options. Attempts at improving treatment should focus on subgroups of refractory disease e.g. by HCT, and on non-CML determinants of survival. After progression to blast crisis, HCT did not provide a significant survival advantage, although a special study showed that most long-term survivors (72\%) were patients who received a transplant. The 10 -year deep molecular remission rates of $70 \%-80 \%$ indicate that the majority of imatinib-treated patients are candidates for treatment discontinuation.

\section{Keywords}

Chronic myeloid leukemia, tyrosine kinase inhibitors, imatinib, treatment strategy, hematopoietic stem cell transplantation, survival.

\section{Introduction}

The only curative treatment for chronic myeloid leukemia (CML) was previously allogenous hemopoietic cell transplantation (HCT) [1]. With the introduction of the tyrosine kinase inhibitor (TKI) imatinib into CML management 15 years ago and the stunning response and survival results, treatment strategy of CML has profoundly changed. TKI became the first line treatment of choice for CML. 


\section{Long term survival}

Meanwhile several long-term observational and randomized studies have matured and 10-year survival outcomes are available. An overview is shown in Table 1. 5-year survival ranges around $90 \%, 10$-year survival around $83 \%$ and 10 year relative survival compared to the general population is more than $90 \%[2,3]$. Similar results have been observed in population based registries $[4,5,6]$. More patients died of comorbidities than of CML [7].

Deep molecular responses are achieved in up to $80 \%$ after 5 to 10 years (Figure 1) suggesting that treatment discontinuation should be possible in these patients [8].

Table 1. Long-term survival rates of CML patients treated with TKI

\begin{tabular}{|c|c|c|c|c|c|c|}
\hline Study & Dose, mg & $\mathrm{n}$ & $\begin{array}{l}\text { Age at diagnosis, } \\
\text { median, years }\end{array}$ & $\begin{array}{l}\text { 5yr survival, } \\
\%\end{array}$ & $\begin{array}{l}\text { 10yr survival, } \\
\%\end{array}$ & $\begin{array}{l}\text { Median } \\
\text { observation } \\
\text { time, years }\end{array}$ \\
\hline $\begin{array}{l}\text { CML-IV HehImann } \\
\text { et al., 2017) [3] }\end{array}$ & IM 400-800 & 1536 & 53 & 90 & 82 & 9.5 \\
\hline $\begin{array}{l}\text { IRIS (Hochhaus et al., } \\
\text { 2017) [9] }\end{array}$ & IM 400 & 553 & 50 & 89 & 83.3 & 10.9 \\
\hline $\begin{array}{l}\text { GIMEMA (Palandri } \\
\text { et al., 2009) [10] }\end{array}$ & IM 400-800 & 559 & 52 & 90 & NA & 5 \\
\hline $\begin{array}{l}\text { Hammersmith (de Lav- } \\
\text { allade et al, 2008) [11] }\end{array}$ & IM 400 & 204 & 46.3 & 83 & NA & 3.2 \\
\hline $\begin{array}{l}\text { PETHEMA (Cervantes } \\
\text { et al., 2010) [12] }\end{array}$ & IM 400 & 210 & 44 & 97.5 & NA & 4.2 \\
\hline $\begin{array}{l}\text { TOPS (Baccarani et al., } \\
2014 \text { [13]) }\end{array}$ & $\begin{array}{l}\text { IM } 400 \\
\text { IM } 800\end{array}$ & $\begin{array}{l}157 \\
319\end{array}$ & $\begin{array}{l}45 \\
48\end{array}$ & $\begin{array}{l}94 \text { (4 years) } \\
93.4 \text { (4 years) }\end{array}$ & NA & $\begin{array}{l}3.5 \\
3.5\end{array}$ \\
\hline $\begin{array}{l}\text { MDACC (Jain et al., } \\
2015 \text { [14]) }\end{array}$ & $\begin{array}{l}\text { IM } 400 \\
\text { IM } 800\end{array}$ & $\begin{array}{l}70 \\
201\end{array}$ & 48.3 & NR & $\begin{array}{l}80 \\
84\end{array}$ & $\begin{array}{l}9.9 \\
(\min .8)\end{array}$ \\
\hline $\begin{array}{l}\text { ILTE8 (CCR only): } \\
\text { Gambacorti-Passerini } \\
\text { et al., 2011 [15] }\end{array}$ & IM NR & 832 & 51 & 98 (6 years) & 95 (8 years) & 5.8 \\
\hline $\begin{array}{l}\text { ENESTnd (Hochhaus } \\
\text { et al., } 2016 \text { [16]) }\end{array}$ & $\begin{array}{l}\text { IM } 400 \\
\text { Nilo } 600 \\
\text { Nilo } 800\end{array}$ & $\begin{array}{l}283 \\
282 \\
281\end{array}$ & $\begin{array}{l}46 \\
47 \\
47\end{array}$ & $\begin{array}{l}92 \\
94 \\
96\end{array}$ & NA & 5.5 \\
\hline $\begin{array}{l}\text { Dasision (Cortes et al., } \\
2016 \text { [17]) }\end{array}$ & $\begin{array}{l}\text { IM } 400 \\
\text { Dasa } 100\end{array}$ & $\begin{array}{l}260 \\
259\end{array}$ & $\begin{array}{l}49 \\
46\end{array}$ & $\begin{array}{l}90 \\
91\end{array}$ & NA & 5 \\
\hline Median (estimate) & & & & 91 & 83 & \\
\hline
\end{tabular}

$\mathrm{NA}=$ not available; $\mathrm{NR}=$ not reported; $\mathrm{yr}=$ year; $\min .=$ minimum; $\mathrm{IM}=$ imatinib; Nilo = Nilotinib; Dasa = Dasatinib; CCR = complete cytogenetic remission.

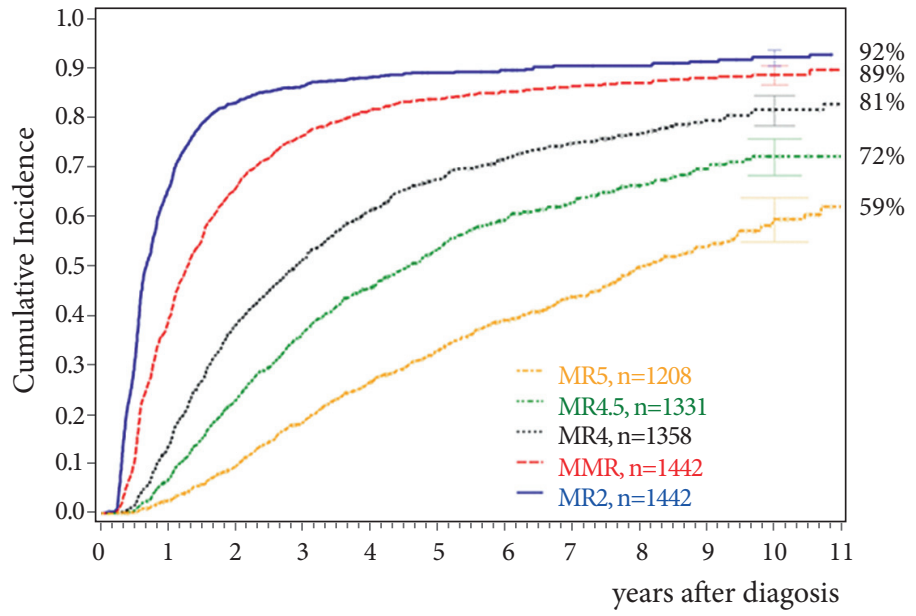

Figure 1. Molecular response achieved by imatinib [8] 


\section{First-line treatment}

Current first-line options with TKI are shown in Table 2. Whereas imatinib has been proven to be safe both at 400 and the faster acting $800 \mathrm{mg}$ daily even after prolonged periods of time, the also faster acting $2^{\text {nd }}$ generation (2G-)TKI require risk assessment due to rare but serious, potentially life threatening adverse drug reactions. As seen from Fig. 2, no survival advantage has been observed with any treatment option $[3,16,17]$. The lower progression rate to blast crisis observed with 2 G-TKI is offset by more deaths due to adverse drug reactions. Variables to be considered in choosing first-line therapy are:

- Risk score;

- Cytogenetics (major-route ACA at diagnosis, high-risk ACA in the course of CML);

- Comorbidities;

- Costs.

The impact of karyotype at diagnosis was demonstrated by Fabarius et al. [18]. Patients with major route ACA which occur in $1-2 \%$ of cases at diagnosis have a much poorer prog-

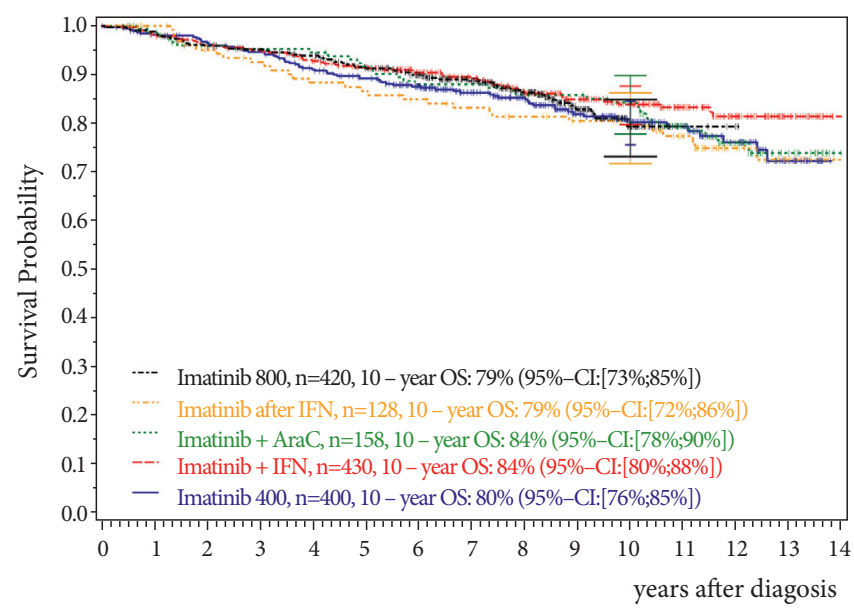

Figure 2. 10-year survival in CML study IV. [3] nosis. Comorbidities do not influence progression of CML, but impact survival more than CML. Generic imatinib has become available recently. It decreases treatment costs at equal efficacy and adds to the advantages of imatinib over 2G-TKI.

\section{Second-line therapy}

$2^{\text {nd }}$-line therapy is needed in cases of refractoriness to imatinib. Table 3 summarizes comparative efficacy and safety of $2^{\text {nd }}$-line treatment options. The variables to be considered for second line therapy are:

- Response milestones (Table 4);

- Adherence to therapy;

- Resistance mutations (Table 5);

- Clonal evolution;

- Intolerance;

- Drug safety;

- Health care setting.

The criteria for assessing TKI-response were proposed by the European LeukemiaNet (ELN) for newly diagnosed CML [13] and are depicted in Table 4. Before changing treatment due to resistance, non-adherence to drug-treatment has to be excluded. Non-adherence has been reported as the most frequent reason for treatment failure [19].

When changing treatment due to confirmed resistance a mutation analysis should be initiated. This can be done simultaneously with changing to the new drug. If the new drug still does not work, the mutation analysis will give a rational basis for selecting the right drug. Table 5 lists the most important mutations and there sensitivity to the currently available TKI.

Adverse TKI reactions have recently been reviewed on behalf of ELN by [20]. Table 6 gives an overview over the most frequently observed adverse TKI reactions.

Table 2. First-line therapy options: Efficacy and safety

\begin{tabular}{|l|l|l|l|l|}
\hline & $\begin{array}{l}\text { Imatinib } \\
\mathbf{4 0 0} \mathbf{m g}\end{array}$ & $\begin{array}{l}\text { IM } 800 \mathrm{mg} \\
\text { tolerability adapted }\end{array}$ & $\begin{array}{l}\text { Nilotinib } \\
2 \times 300 \mathrm{mg}\end{array}$ & $\begin{array}{l}\text { Dasatinib } \\
100 \mathrm{mg}\end{array}$ \\
\hline Efficacy & & acts faster & $\begin{array}{l}\text { acts faster, } \\
\text { less early progressions }\end{array}$ & $\begin{array}{l}\text { acts faster, } \\
\text { less early progressions }\end{array}$ \\
\hline Safety & Safe & Safe & assess risks & assess risks \\
\hline Survival & \multicolumn{2}{|c|}{$82-86 \%$ after 10 years } & \multicolumn{2}{|c}{$91 \%$ after 5 years } \\
\hline
\end{tabular}

Note: No survival advantage with any therapy option. 
Table 3. Second line treatment options: Efficacy and safety

\begin{tabular}{|l|l|l|l|l|l|}
\hline & Dasatinib & Nilotinib & Bosutinib & Ponatinib & HCT \\
\hline Efficacy & $\begin{array}{l}\text { Nilotinib resistance } \\
\text { mutations } \\
\text { AP, BC }\end{array}$ & $\begin{array}{l}\text { Dasatinib resistance } \\
\text { mutations } \\
\text { Renal failure }\end{array}$ & $\begin{array}{l}\text { After failure } \\
\text { of 2 TKI }\end{array}$ & $\begin{array}{l}\text { T315I } \\
\text { AP, BC }\end{array}$ & $\begin{array}{l}\text { CE } \\
\text { AP, BC }\end{array}$ \\
\hline Safety & $\begin{array}{l}\text { No pulmonary risks } \\
\text { N.B.: } \\
\text { infections }\end{array}$ & $\begin{array}{l}\text { No CV risks } \\
\text { N.B.: diabetes, liver } \\
\text { disease }\end{array}$ & N.B.: Gl-toxicity & No CV risks & $\begin{array}{l}\text { No HCT contra- } \\
\text { indications }\end{array}$ \\
\hline
\end{tabular}

Note: No survival advantage with any treatment option.

$\mathrm{AP}=$ Accelerated phase, $\mathrm{BC}=$ Blast crisis, $\mathrm{CE}=$ Clonal evolution, $\mathrm{CV}=$ Cardiovaskular, $\mathrm{GI}=$ Gastrointestinal, $\mathrm{HCT}=\mathrm{Hemo}-$ poietic cell transplantation

Table 4. ELN response milestones for newly diagnosed CML [13]

\begin{tabular}{|c|c|c|c|}
\hline Time: & Optimal Response & Warning & Failure \\
\hline 3 months & $\begin{array}{l}\text { BCR/ABL1 } \leq 10 \% \\
\text { Ph+ cells } \leq 35 \% \text { (PCyR) }\end{array}$ & $\begin{array}{l}\text { BCR/ABL1 >10\% } \\
\text { Ph+ cells 36-95\% }\end{array}$ & $\begin{array}{l}\text { No CHR. } \\
\text { Ph+ cells >95\% }\end{array}$ \\
\hline 6 months & $\begin{array}{l}\text { BCR/ABL1 <l\% } \\
\mathrm{Ph}^{+} \text {cells 0\% (CCyR) }\end{array}$ & $\begin{array}{l}\text { BCR/ABL1 1-10\% } \\
\text { Ph+ cells 1-35\% }\end{array}$ & $\begin{array}{l}\text { BCR/ABL1 >10\% } \\
\text { Ph+ cells >35\% }\end{array}$ \\
\hline 12 months & BCR/ABL1 $\leq 0.1 \%$ (MMR) & BCR/ABL1 $0.1-1 \%$ & $\begin{array}{l}\mathrm{BCR} / \mathrm{ABL1}>1 \% \\
\mathrm{Ph}+\text { cells }>0 \%\end{array}$ \\
\hline Thereafter & $\begin{array}{l}\text { Major Molecular Response [MMR] } \\
\text { or better; Tolerating the drug; good } \\
\text { adherence; monitored every } 3 \text { mos }\end{array}$ & $\begin{array}{l}-7 \text { or del(7q) } \\
\text { in Ph- cells }\end{array}$ & $\begin{array}{l}\text { Loss of CHR or CCyR; confirmed } \\
\text { loss of MMR. ABLI mutations. } \\
\text { New chromosome abnormalities }\end{array}$ \\
\hline
\end{tabular}

Table 5. Impact of TKI resistance mutations of the BCR/ABL kinase domain [21] (permission of reproduction granted by $M$. Deininger)

\begin{tabular}{|c|c|c|c|c|c|}
\hline \multirow{2}{*}{ Mutation } & \multicolumn{5}{|c|}{$\mathrm{IC}_{50}$-fold increase relative to $\mathrm{WT}(\mathrm{W}=1)$} \\
\hline & Imatinib & Bosutinib & Dasatinib & Nilotinib & Ponatinib \\
\hline M244V & 0.9 & 0.9 & 2.0 & 1.2 & 3.2 \\
\hline L248R & 14.6 & 22.9 & 12.5 & 30.2 & 6.2 \\
\hline L248V & 3.5 & 3.5 & 5.1 & 2.8 & 3.4 \\
\hline G250E & 6.9 & 4.3 & 4.4 & 4.6 & 6.0 \\
\hline Q252H & 1.4 & 0.8 & 3.1 & 2.6 & 6.1 \\
\hline Y253F & 3.6 & 1.0 & 1.6 & 3.2 & 3.7 \\
\hline Y253H & 8.7 & 0.6 & 2.6 & 36.8 & 2.6 \\
\hline E255K & 6.0 & 9.5 & 5.6 & 6.7 & 8.7 \\
\hline E255V & 17.0 & 5.5 & 3.4 & 10.3 & 12.9 \\
\hline D276G & 2.2 & 0.6 & 1.4 & 2.0 & 2.1 \\
\hline E279K & 3.6 & 1.0 & 1.6 & 2.0 & 3.0 \\
\hline E292L & 0.7 & 1.1 & 1.3 & 1.8 & 2.0 \\
\hline V299L & 1.5 & 26.1 & 8.7 & 1.3 & 0.6 \\
\hline T315A & 1.7 & 6.0 & 58.9 & 2.7 & 0.4 \\
\hline T315I & 17.5 & 45.4 & 75.0 & 39.4 & 3.0 \\
\hline T315V & 12.2 & 29.3 & 738.8 & 57.0 & 2.1 \\
\hline F317L & 2.6 & 2.4 & 4.5 & 2.2 & 0.7 \\
\hline F317R & 2.3 & 33.5 & 114.8 & 2.3 & 4.9 \\
\hline F317V & 0.4 & 11.5 & 21.3 & 0.5 & 2.3 \\
\hline M343T & 1.2 & 1.1 & 0.9 & 0.8 & 0.9 \\
\hline M351T & 1.8 & 0.7 & 0.9 & 0.4 & 1.2 \\
\hline F359I & 6.0 & 2.9 & 3.0 & 16.3 & 2.9 \\
\hline F359V & 2.9 & 0.9 & 1.5 & 5.2 & 4.4 \\
\hline L384M & 1.3 & 0.5 & 2.2 & 2.3 & 2.2 \\
\hline H396P & 2.4 & 0.4 & 1.1 & 2.4 & 1.4 \\
\hline H396R & 3.9 & 0.8 & 1.6 & 3.1 & 5.9 \\
\hline F486S & 8.1 & 2.3 & 3.0 & 1.9 & 2.1 \\
\hline $\begin{array}{l}\text { L248R + } \\
\text { F359I }\end{array}$ & 11.7 & 39.3 & 13.7 & 96.2 & 17.7 \\
\hline
\end{tabular}

\begin{tabular}{|l|l|}
\hline Sensitive & $\begin{array}{l}<2 \text {-fold } \\
\text { difference }\end{array}$ \\
\hline $\begin{array}{l}\text { Moderately } \\
\text { sensitive }\end{array}$ & $\begin{array}{l}\text { 2.1-to 4-fold } \\
\text { difference }\end{array}$ \\
\hline Resistant & $\begin{array}{l}4.1 \text {-to } 10 \text {-fold } \\
\text { difference }\end{array}$ \\
\hline Highly resistant & $\begin{array}{l}>10 \text {-fold } \\
\text { difference }\end{array}$ \\
\hline
\end{tabular}


REVIEW ARTICLES

Table 6. Adverse TKI reactions: types and severity [20]

\begin{tabular}{|l|l|l|l|l|l|}
\hline & Imatinib & Nilotinib & Dasatinib & Bosutinib & Ponatinib \\
\hline Myelosuppression & ++ & + & +++ & + & ++ \\
\hline Fluid retention & ++ & - & +++ & - & - \\
\hline Rash & + & ++ & - & - & +++ \\
\hline Diarrhea & + & + & + & - & + \\
\hline C Glucose / Cholesterol & - & ++ & - & - & - \\
\hline Vascular occlusion & - & ++ & + & $?$ & +++ \\
\hline Renal insufficiency & + & - & ++ & $?$ \\
\hline
\end{tabular}

\section{Studies IIIA and IV}

CML study IIIA is a geneticly randomized study comparing allogenous HCT with best available drug treatment. It recruited 662 patients, randomized 427 eligible patients (family donor available vs not available) and was published after a median observation time of 12.1 years [15]. The key result was equivalence of outcome for low risk patients after transplantion, if performed within one year of diagnosis, and imatinib.
CML study IV is a randomized 5-arm treatment optimization study to explore whether treatment with imatinib $400 \mathrm{mg}$ can be improved by doubling the dose, combining imatinib with cytarabine or interferon $\alpha$ (IFN) or applying imatinib after IFN failure. 1551 newly diagnosed patients in chronic phase where recruited and the study published after a median observation time of 9.5 years [3]. The key outcome was no superiority of survival of any treatment option (Fig. 2) in spite of significantly faster responses with imatinib $800 \mathrm{mg}$ and the recognition of determinants of survival independent of treatment by multivariate analysis (Table 7).

Table 7. Determinants of survival by multivariate analysis ( $n=1252)$

\begin{tabular}{|l|l|l|l|}
\hline Variable & & Hazard ratio & p-value \\
\hline Therapy & IM-after-IFN-failure vs. IM 400 & 1.334 & 0.256 \\
& IM 800 vs. IM 400 & 1.033 & 0.875 \\
& IM + cytarabine vs. IM 400 & 1.170 & 0.519 \\
& IM + IFN vs. IM 400 & 0.933 & 0.727 \\
\hline Risk-score & Low vs. High risk & 0.459 & $<0.001$ \\
& Intermediate vs. High Risk & 1.062 & 0.770 \\
\hline Center effect & Academic vs. community hospital & 1.515 & 0.021 \\
& Academic vs. office-based center & 1.768 & 0.004 \\
\hline Comorbidity \\
(Charlson index) & Per point (age not considered) & 1.518 & $<0.001$ \\
\hline Gender & Male vs. female & 1.199 & 0.240 \\
\hline Transcript type & b2a2 vs. b3a2 & 1.092 & 0.574 \\
& b2a2 + b3a2 vs. b3a2 & 1.171 & 0.447 \\
\hline Smoking habit & Smoker vs. non-smoker & 1.728 & 0.001 \\
\hline Karyotype & Major-Route ACA vs. & 6.137 & $<0.001$ \\
\hline
\end{tabular}

Note: ${ }^{*}$, age considered by Risk-score. IM = Imatinib, IFN = interferon $\alpha, \mathrm{ACA}=$ additional chromosomal aberration 
A comparison of long-term survival after HCT or drug treatment showed that low risk patients had similar survival with both options (Fig. 3) [22, 23].

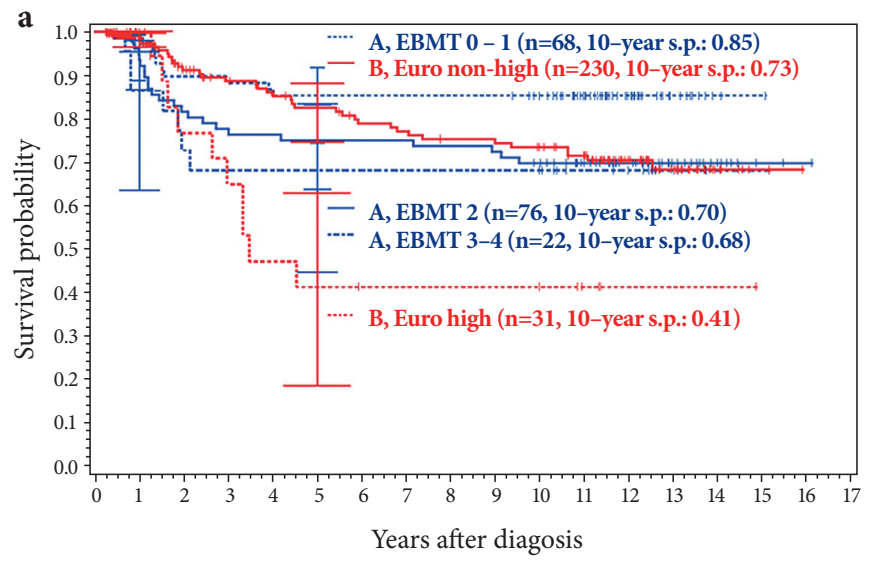

Figure 3. HCT (Group A in blue) vs. drug treatment (Group B in red) by transplant (EBMT score) - and disease- risks (EURO score) [22]

After progression to blast crisis HCT did not provide a significant survival advantage (Fig. 4), although long-term observations of 699 blast crises from the German CML studies showed that most long-term survivors (72\%) were patients who received a transplant [24].

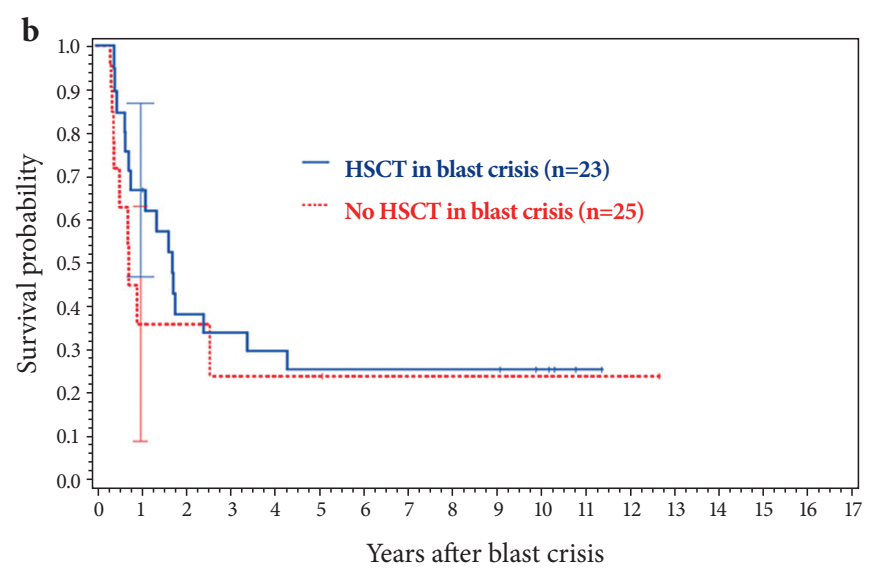

Figure 4. Effects of hematopoietic stem cell transplantation upon survival of CML patients with blast crisis [22]

\section{Conclusion}

- Imatinib $400 \mathrm{mg}$ provides close to normal life expectancy in chronic-phase CML patients.

- Survival is independent of time to response.

- Outcome of CML is currently more determined by disease and patients' factors e.g. comorbidities and smoking, and by center effects than by initial treatment selection.

- In low risk patients survival after imatinib and transplantation may be similar.

- Attempts at improving treatment should focus on subgroups of refractory disease e.g. by HCT, and on non-CML determinants of survival.

- The 10-year deep molecular remission rates of 70\%-80\% indicate that the majority of imatinib treated patients are candidates for treatment discontinuation.

\section{Conflict of interest}

The author has no conflicts of interest to declare.

\section{References}

1. Goldman JM. Chronic myeloid leukemia: reversing the chronic phase. J Clin Oncol. 2010;28(3):363-365.

2. Pfirrmann M, Baccarani M, Saussele S, Guilhot J, Cervantes F, Ossenkoppele G, Hoffmann VS, Castagnetti F, Hasford J, Hehlmann R, Simonsson B. Prognosis of long-term survival considering disease-specific death in patients with chronic myeloid leukemia. Leukemia. 2016;30(1):48-56.

3. Hehlmann R, Lauseker M, Saußele S, Pfirrmann M, Krause S, Kolb HJ, Neubauer A, Hossfeld DK, Nerl C, Gratwohl A, Baerlocher GM, Heim D, Brümmendorf TH, Fabarius A, Haferlach C, Schlegelberger B, Müller MC, Jeromin S, Proetel U, Kohlbrenner K, Voskanyan A, Rinaldetti S, Seifarth W, Spieß B, Balleisen L, Goebeler MC, Hänel M, Ho A, Dengler J, Falge C, Kanz L, Kremers S, Burchert A, Kneba M, Stegelmann F, Köhne CA, Lindemann HW, Waller CF, Pfreundschuh M, Spiekermann K, Berdel WE, Müller L, Edinger M, Mayer J, Beelen DW, Bentz M, Link H, Hertenstein B, Fuchs R, Wernli M, Schlegel F, Schlag R, de Wit M, Trümper L, Hebart H, Hahn M, Thomalla J, Scheid C, Schafhausen P, Verbeek W, Eckart MJ, Gassmann W, Pezzutto A, Schenk M, Brossart P, Geer T, Bildat S, Schäfer E, Hochhaus A, Hasford J. Assessment of imatinib as first-line treatment of chronic myeloid leukemia: 10-year survival results of the randomized CML study IV and impact of non-CML determinants. Leukemia. 2017;31(11):2398-2406.

4. Höglund M, Sandin F, Hellström K, Björeman M, Björkholm M, Brune M, Dreimane A, Ekblom M, Lehmann S, Ljungman P, Malm C, Markevärn B, Myhr-Eriksson K, Ohm L, Olsson-Strömberg U, Själander A, Wadenvik H, Simonsson B, Stenke L, Richter J. Tyrosine kinase inhibitor usage, treatment outcome, and prognostic scores in CML: report from the population-based Swedish CML registry. Blood. 2013;122(7):1284-1292.

5. Thielen N, Visser O, Ossenkoppele G, Janssen J. Chronic myeloid leukemia in the Netherlands: a population-based study on incidence, treatment, and survival in 3585 patients from 1989 to 2012. Eur J Haematol. 2016;97(2):145-154.

6. Bower H, Björkholm M, Dickmann P, Höglund M, Lambert PC, Andersson TM. Life expectancy of patients with CML approaches the life expectancy of the general population. J Clin Oncol 2016;34(24): 2851-2858.

7. Saussele S, Krauss MP, Hehlmann R, Lauseker M, Proetel U, Kalmanti L, Hanfstein B, Fabarius A, Kraemer D, Berdel WE, Bentz M, Staib P, de Wit M, Wernli M, Zettl F, Hebart HF, Hahn M, Heymanns J, Schmidt-Wolf I, Schmitz N, Eckart MJ, Gassmann W, Bartholomäus A, Pezzutto A, Leibundgut EO, Heim D, Krause SW, Burchert A, Hofmann WK, Hasford J, Hochhaus A, Pfirrmann M, Müller MC; Schweizerische Arbeitsgemeinschaft für Klinische Krebsforschung and the German CML Study Group. Impact of comorbidities on overall survival in patients with chronic myeloid leukemia: results of the randomized CML study IV. Blood 2015;126(1):42-49. 
8. Kalmanti L, Saussele S, Lauseker M, Müller MC, Dietz CT, Heinrich L, Hanfstein B, Proetel U, Fabarius A, Krause SW, Rinaldetti S, Dengler J, Falge C, Oppliger-Leibundgut E, Burchert A, Neubauer A, Kanz L, Stegelmann F, Pfreundschuh M, Spiekermann K, Scheid C, Pfirrmann M, Hochhaus A, Hasford J, Hehlmann R. Safety and efficacy of imatinib in CML over a period of 10 years: data from the randomized CML-study IV.Leukemia. 2015; 29(5):1123-1132.

9. Hochhaus A, Larson RA, Guilhot F, Radich JP, Branford S, Hughes TP, Baccarani M, Deininger MW, Cervantes F, Fujihara S, Ortmann CE, Menssen HD, Kantarjian H, O’Brien SG, Druker BJ; IRIS Investigators. Long-Term Outcomes of Imatinib Treatment for Chronic Myeloid Leukemia. N Engl J Med. 2017;376(10):917-927.

10. Palandri F, Castagnetti F, Alimena G, Testoni N, Breccia M, Luatti S, Rege-Cambrin G, Stagno F, Specchia G, Martino B, Levato L, Merante S, Liberati AM, Pane F, Saglio G, Alberti D, Martinelli G, Baccarani M, Rosti G. The long-term durability of cytogenetic responses in patients with accelerated phase chronic myeloid leukemia treated with imatinib 600 mg: the GIMEMA CML Working Party experience after a 7-year follow-up. Haematologica. 2009;94(2):205-12.

11. de Lavallade H, Apperley JF, Khorashad JS, Milojkovic D, Reid AG, Bua M, Szydlo R, Olavarria E, Kaeda J, Goldman JM, Marin D. Imatinib for newly diagnosed patients with chronic myeloid leukemia: incidence of sustained responses in an intention-to-treat analysis. J Clin Oncol. 2008;26(20):3358-3363.

12. Cervantes F, López-Garrido P, Montero MI, Jonte F, Martínez J, Hernández-Boluda JC, Calbacho M, Sureda A, Pérez-Rus G, Nieto JB, Pérez-López C, Román-Gómez J, González M, Pereira A, Colomer D. Early intervention during imatinib therapy in patients with newly diagnosed chronic-phase chronic myeloid leukemia: a study of the Spanish PETHEMA group. Haematologica. 2010 Aug;95(8):13171324.

13. Baccarani M, Druker BJ, Branford S, Kim DW, Pane F, Mongay L, Mone M, Ortmann CE, Kantarjian HM, Radich JP, Hughes TP, Cortes JE, Guilhot F. Long-term response to imatinib is not affected by the initial dose in patients with Philadelphia chromosome-positive chronic myeloid leukemia in chronic phase: final update from the Tyrosine Kinase Inhibitor Optimization and Selectivity (TOPS) study. Int J Hematol. 2014;99(5):616-624.

14. Jain P, Kantarjian H, Alattar ML, Jabbour E, Sasaki K, Nogueras Gonzalez G, Dellasala S, Pierce S, Verstovsek S, Wierda W, Borthakur G, Ravandi F, O'Brien S, Cortes J. Long-term molecular and cytogenetic response and survival outcomes with imatinib $400 \mathrm{mg}$, imatinib $800 \mathrm{mg}$, dasatinib, and nilotinib in patients with chronic-phase chronic myeloid leukaemia: retrospective analysis of patient data from five clinical trials. Lancet Haematol. 2015;2(3):e118-28.

15. Gambacorti-Passerini C, Antolini L, Mahon FX, Guilhot F, Deininger M, Fava C, Nagler A, Della Casa CM, Morra E, Abruzzese E, D’Emilio A, Stagno F, le Coutre P, Hurtado-Monroy R, Santini V, Martino B, Pane F, Piccin A, Giraldo P, Assouline S, Durosinmi MA, Leeksma O, Pogliani EM,
Puttini M, Jang E, Reiffers J, Piazza, Valsecchi MG, Kim DW. Multicenter independent assessment of outcomes in chronic myeloid leukemia patients treated with imatinib. J Natl Cancer Inst. 2011;103(7):553-561.

16. Hochhaus A, Saglio G, Hughes TP, Larson RA, Kim DW, Issaragrisil S, le Coutre PD, Etienne G, Dorlhiac-Llacer PE, Clark RE, Flinn IW, Nakamae H, Donohue B, Deng W, Dalal D, Menssen HD, Kantarjian HM. Long-term benefits and risks of frontline nilotinib vs imatinib for chronic myeloid leukemia in chronic phase: 5-year update of the randomized ENESTnd trial. Leukemia. 2016 May;30(5):1044-1054.

17. Cortes JE, Saglio G, Kantarjian HM, Baccarani M, Mayer J, Boqué C, Shah NP, Chuah C, Casanova L, Bradley-Garelik B, Manos G, Hochhaus A.Final 5-Year Study Results of DASISION: The Dasatinib Versus Imatinib Study in Treatment-Naïve Chronic Myeloid Leukemia Patients Trial. J Clin Oncol. 2016;34(20):2333-2340.

18. Fabarius A, Kalmanti L, Dietz CT, Lauseker M, Rinaldetti S, Haferlach C, Göhring G, Schlegelberger B, Jotterand M, Hanfstein B, Seifarth W, Hänel M, Köhne CH, Lindemann HW, Berdel WE, Staib P, Müller MC, Proetel U, Balleisen L, Goebeler ME, Dengler J, Falge C, Kanz L, Burchert A, Kneba M, Stegelmann F, Pfreundschuh M, Waller CF, Spiekermann K, Brümmendorf TH, Edinger M, Hofmann WK, Pfirrmann M, Hasford J, Krause S, Hochhaus A, Saußele S, Hehlmann R; SAKK and the German CML Study Group. Impact of unbalanced minor route versus major route karyotypes at diagnosis on prognosis of CML. Ann Hematol. 2015;94(12):2015-2024.

19. Noens L, Hensen M, Kucmin-Bemelmans I, Lofgren C, Gilloteau I, Vrijens B. Measurement of adherence to BCR$\mathrm{ABL}$ inhibitor therapy in chronic myeloid leukemia: current situation and future challenges. Haematologica. 2014; 99(3):437-447.

20. Steegmann JL, Baccarani M, Breccia M, Casado LF, García-Gutiérrez V, Hochhaus A, Kim DW, Kim TD, Khoury HJ, Le Coutre P, Mayer J, Milojkovic D, Porkka K, Rea D, Rosti G, Saussele S, Hehlmann R, Clark RE. European LeukemiaNet recommendations for the management and avoidance of adverse events of treatment in chronic myeloid leukaemia. Leukemia. 2016;30(8):1648-1671.

21. Eiring AM, Deininger MW. Individualizing kinase-targeted cancer therapy: the paradigm of chronic myeloid leukemia. Genome Biol. 2014 Sep 17;15(9):461. doi: 10.1186/ s13059-014-0461-8.

22. Gratwohl A, Pfirrmann M, Zander A, Kröger N, Beelen D, Novotny J, Nerl C, Scheid C, Spiekermann K, Mayer J, Sayer HG, Falge C, Bunjes D, Döhner H, Ganser A, SchmidtWolf I, Schwerdtfeger R, Baurmann H, Kuse R, Schmitz N, Wehmeier A, Fischer JT, Ho AD, Wilhelm M, Goebeler ME, Lindemann HW, Bormann M, Hertenstein B, Schlimok G, Baerlocher GM, Aul C, Pfreundschuh M, Fabian M, Staib P, Edinger M, Schatz M, Fauser A, Arnold R, Kindler T, Wulf G, Rosselet A, Hellmann A, Schäfer E, Prümmer O, Schenk M, Hasford J, Heimpel H, Hossfeld DK, Kolb HJ, Büsche G, Haferlach C, Schnittger S, Müller MC, Reiter A, Berger U, Saußele S, Hochhaus A, Hehlmann R; SAKK; German CML 
Study Group. Long-term outcome of patients with newly diagnosed chronic myeloid leukemia: a randomized comparison of stem cell transplantation with drug treatment. Leukemia. 2016 Mar;30(3):562-569.

23. Saussele S, Lauseker M, Müller MC, Gratwohl A, Beelen D, Bunjes DW et al. Allogeneic hematopoietic stem cell transplantation (HSCT) in the imatinib-era: update on the survival outcome following allogeneic HSCT after imatinib failure; results of the German CML study IV. Blood. 2014;124 (ASH Meeting abstract No. 2567).

24. Hehlmann R, Saussele S. Treatment of chronic myeloid leukemia in blast crisis. Haematologica 2008; 93:1765-1769.

\title{
Долгосрочная выживаемость при хроническом миелоидном лейкозе при лечении иматинибом или трансплантации гемопоэтических клеток в качестве первой линии терапии: сравнение исходов по данным исследований CML IIIA и IV
}

\author{
Рюдигер Хельманн \\ Медицинский факультет Маннгейма, Гейдельбергский университет, Германия
}

\section{Резюме}

Стратегия лечения хронического миелолейкоза (ХМЛ) сильно изменилась после внедрения иматиниба - ингибитора тирозинкиназы (ИТК). Эти препараты стали часто применяться для первой линии лечения при ХМЛ вместо аллогенной трансплантации гемопоэтических стволовых клеток (ТГСК). При выборе ИТК в качестве терапии первой линии учитывают следующие факторы: оценка риска для больного по принятой шкале, цитогенетические маркеры со значимыми дополнительными хромосомными аномалиями (ДХА) при постановке диагноза, а также ДХА высокого риска в процессе развития ХМЛ, сопутствующие заболевания, расходы на лечение. В случаях отсутствия ответа на иматиниб, рассматриваются возможности 2-й линии терапии, с учетом показателей клинического ответа на лечение, соблюдения режима лечения, мутаций, ведущих к лекарственной устойчивости, клональной эволюции лейкоза, непереносимости данного лечения, типа лечебного учреждения.

В программе CML IV, рандомизированном исследовании, направленном на оптимизацию дозы иматиниба и эффектов сочетанной терапии иматиниба с цитарабином или интерфероном $\alpha$, участвовали 1551 свежевыявленных пациентов в хронической фазе ХМЛ. Основным результатом было отсутствие какого-либо преимущества любого из применявшихся методов лечения. Иматиниб в дозе 400 мг обеспечи- вает близкую к норме ожидаемую продолжительность жизни у больных с ХМЛ в хронической фазе. Выживаемость пациентов независима от времени ответа. Клинические исходы ХМЛ теперь определяются скорее факторами заболевания и особенностями пациентов, например - сопутствующими заболеваниями и курением, а также подходом конкретных медицинских центров, нежели выбором тактики первичного лечения. Сравнение долгосрочной выживаемости после ТГСК или лечения иматинибом показало, что больные из группы низкого риска имели сходную выживаемость при обоих вариантах лечения. Попытки улучшения терапии, например, с применением ТГСК должны быть сосредоточены на группах рефрактерных пациентов, а также на показателях выживаемости, не связанных с ХМЛ. После прогрессии в бластный криз ТГСК не обеспечивает существенного преимущества в выживаемости, хотя специальное исследование показало, что наиболее долгоживущие пациенты (72\%) были леченными посредством ТГСК. $70 \%$ - $80 \%$ частота 10 -летней глубокой молекулярной ремиссии указывает на то, что большинство больных, леченых иматинибом, являя. тся кандидатами на прекращение терапии.

\section{Ключевые слова}

Хронический миелоидный лейкоз, ингибиторы тирозинкиназы, иматиниб, стратегия лечения, трансплантация гемопоэтических клеток, выживаемость. 